\title{
Psicobiología del síndrome de Down
}

\author{
Angela Loeches Alonso, Jaime Iglesias Dorado, \\ Fernando Carvajal Molina \\ Universidad Autónoma de Madrid
}

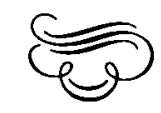

Resumen

En este articulo revisamos los conocimientos que existen en la actualidad sobre el sindrome de Down, atendiendo a todos aquellos aspectos que, por su relevancia, facilitan una mejor comprensión de esta cromosomopatía. Después de una breve reseña bistórica, y tras definir lo que se entiende por sindrome de Down, pasamos a ocuparmos de la alteración genética que lo determina, así como de los factores que parecen estar relacionados con su origen. A continuación estudiamos las alteraciones específicas que acompañan a este sindrome, considerando principalmente los aspectos morfológicos externos, el problema de la hipotonía muscular y otros trastomos biomédicos, el retraso mental y las diversas anomalias neuroanatómicas y neurofisiológicas que presentan los sujetos afectados. Por último, indicamos las nuevas perspectivas que se abren en el estudio de esta alteración genética.

Palabras clave: Cromosomopatías, Síndrome de Down, Mongolismo, Retraso mental.

\section{Abstract}

In this paper we have reviewed the current knowledges about Down's syndrome, considering all those aspects that are important for understanding this chromosome imbalance. After a short bistorical note and baving defined what can be understood as Down's syndrome, we bave attended to the genetic disorder that causes it, and to other factors that seem to be related to its origin. Afterwards, we have studied the specific anomalies associated to this syndrome, principally the external morphological aspects, the deficit in the muscular tone and otbers biomedical disorders, the mental retardation and the neuroanatomical and neurophysiological disturbances that suffer the affected subjects. Lastly, we point out the new perspectives in the study of this genetic disorder.

Key words: Chromosomal dirsorder, Down's syndrome, Mongolism, Mental retardation.

Dirección del autor: Universidad Autónoma de Madrid. Departamento de Psicología Biológica y de la Salud. Facultad de Psicología. Canto Blanco. 28049 Madrid. 


\section{INTRODUCCION HISTORICA Y DEFINICION}

El descubrimiento del síndrome de Down como entidad clínica específica se atribuye al médico inglés Langdon Down, quien en 1866 publicó un artículo, para destacar la existencia en Europa de un conjunto de retrasados mentales caracterizados por presentar una morfología facial peculiar en relación con los sujetos normales. No obstante, con antelación a esta fecha ya se habían publicado descripciones aisladas acerca de ciertos pacientes que, además de diversas patologías, presentaban las peculiaridades morfológicas distintivas de lo que más tarde recibiría el nombre, entre otros, de síndrome de Down (véanse, por ejemplo, las descripciones de casos clínicos realizadas por Esquirol, 1838, y Seguin, 1846).

Desde finales del siglo XIX y primeros años del siglo XX, sobresale la aparición de un número creciente de artículos sobre el síndrome de Down en las publicaciones científicas de todo el mundo. En su mayoría, se trataba de estudios descriptivos sobre diferentes aspectos del propio síndrome o de variables supuestamente relacionadas con éste. Algunos trabajos perteneciente a este período son el de Jones (1890), que trataba sobre el cerebro de los sujetos con síndrome de Down; el de Oliver (1891), quien describió la morfología de los ojos y dedos de dichos sujetos, o el de Turpin y Caratzali (1934), quienes pusieron de relieve la relación de la edad materna con el síndrome de Down.

Los autores se interesaron por determinar desde un primer momento la causa o posibles causas de esta alteración. Entre todas las explicaciones propuestas originalmente, la que tuvo mayor impacto sobre la comunidad científica de la época, aun no siendo la más convincente, fue la que el propio $\mathrm{Dr}$. Down postuló cuando identificó el síndrome. La explicación propuesta por Down se basaba principalmente en las peculiaridades del rostro de un conjunto de retrasados mentales, quienes presentaban, entre otros rasgos, una nariz chata, los pómulos pronunciados, unos ojos rasgados y, en algunos casos, la piel ligeramente amarillenta. La supuesta similitud de estos rasgos con la morfología facial de determinadas poblaciones de individuos orientales, una interpretación errónea de la teoría de la evolución de Darwin y el pensamiento racista de la época llevaron al descubridor de esta alteración biológica a explicarla como un ejemplo de «regresión evolutiva». Así, Down sugirió que la anomalía observada podría deberse a un proceso de degeneración de la raza blanca (considerada en el siglo XIX como la más evolucionada) hacia grupos étnicos que ocupaban lugares más bajos en las clasificaciones raciales de la época, como eran en este caso los mongoles. Gould, en la traducción castellana de 1983 de su obra El pulgar del panda, cita textualmente las siguiente palabras del Dr. Down, que son el mejor testimonio para ilustrar sus conclusiones:

«Un gran número de idiotas congénitos son mongoles típicos. Tan marcado es esto, que, al ponerlos juntos, resulta difícil creer que los especímenes comparados no sean hijos de los mismos padres». (pág. 174).

Aunque las ideas del Dr. Down no tuvieron una gran acogida, los términos «mongol» $\mathrm{y}$ «mongólico» llegaron a ser de uso común para hacer referencia a los individuos que padecían esta alteración. Sin embargo, por una parte, la mayoría de los autores dudaron desde un primer momento que los pacientes así llamados tuvieran en realidad alguna relación con los individuos mongoles; por otra parte, el hecho de que posteriormente se informase de casos afectados de 
esta patología en poblaciones distintas a la europea invalidó por completo la teoría de Down.

Las investigaciones que permitieron comprender definitivamente el motivo de esta alteración no aparecieron hasta el segundo decenio del siglo XX. Hasta entonces, las hipótesis causales propuestas por los distintos autores fueron muy divesas. Así, entre las posibles causas del síndrome de Down llegaron a considerarse las siguientes: el padecimiento por parte de alguno de los progenitores de sífilis (Sutherland, 1899) o de tuberculosis (Shuttleworth, 1906); el parentesco con algún individuo epiléptico, «loco» o retrasado mental (Tredgold, 1908; Caldecott, 1909); el alcoholismo paterno (Cafferata, 1909); la edad de la madre y el número de embarazos previsos al del sujeto afectado (Shuttleworth, 1909); la regresión a formas de conducta de primates no humanos (Crookshank, 1924); el tamaño reducido del saco amniótico materno (Jansen, 1921); y, por último, el hipertiroidismo fetal (Clark, 1929). Con posterioridad, podemos decir que la mayoría de estas explicaciones se refieren a aspectos que nada tienen que ver con el síndrome de Down y que ninguna de ellas se aproxima a su verdadera causa.

Tan sólo cuando los autores llegaron a centrarse en el estudio de casos de gemelos monocigóticos y dicigóticos que padecían el síndrome de Down (Halbertsma, 1923; Reuben y Klein, 1926, y Keay, 1958), casos de madres afectadas con algún hijo que también presentaba la alteración (Lelong y cols., 1949; Rehn y Thomas, 1957) y casos de familias con más de un individuo afectado (Penrose, 1951, 1953), se comenzó a pensar que alguna anomalía genética podría estar implicada en el orígen del síndrome, con independencia de otros factores. Ahondando en esta posibilidad, un grupo de investigadores franceses, encabezados por Lejeune, Turpin y Gautier, descubrieron en 1959 que los individuos que padecían esta alteración presentaban en todas o algunas de sus células un número total de 47 cromosomas, cuando se sabía desde 1956 que la dotación normal en el hombre era de 46, distribuidos en 23 pares. En el caso particular de los sujetos afectados del síndrome de Down, uno de los pares, en concreto el 21, era extranumerario, al contener tres cromosomas en lugar de dos. Hoy sabemos con seguridad que los investigadores franceses estaban en lo cierto, ya que la causa de la alteración no es otra que la que ellos determinaron hace casi treinta años. En el apartado siguiente decribiremos con más detalle las bases genéticas de esta anomalía, así como los distintos mecanismos que posibilitan la aparición de un cromosoma extra en la dotación genética total de un individuo.

El descubrimiento de la cuasa del síndrome de Down permitió abordar el estudio de este fenómeno de una forma más racional, dando lugar a un cambio radical de las coordenadas en las que quedaba encuadrado. Este cambio se reflejó en aspectos tan elementales como la búsqueda de términos apropiados para referirse a los sujetos afectados por esta patología que no tuviesen que ver con las concepciones inadecuadas antes mencionadas. A este respecto, la comunidad científica propuso sustituir los términos de «mongol», «mongólico» $\mathrm{y}$ «mongolismo» por otros como «síndrome de Down», recogiendo así el nombre de su descubridor, o «trisomía 21", haciendo en este caso referencia a la alteración presente en el par de cromosomas 21. Aunque estos cambios de nomenclatura no han quedado aún establecidos definitivamente, nosotros nos sumamos a las intenciones actuales y utilizaremos a partir de ahora los términos «síndrome de Down» o «trisomía 21» para referirnos a los sujetos afectados, así como a la propia alteración. 
Los estudios científicos actuales sobre el síndrome de Down tienen por objeto un mayor esclarecimiento de los aspectos genéticos, bioquímicos, fisiológicos, neurológicos y comportamentales asociados a esta anomalía, así que son especialistas de muy diversas disciplinas los que se ocupan hoy día en este campo de investigación. El interés que ha adquirido el tema en los últimos años ha permitido un crecimiento rápido de los conocimientos sobre este fenómeno, dando lugar a un campo de trabajo con entidad propia, que atrae cada vez más la atención de la comunidad científica.

\section{BASES GENETICAS}

Como ya se ha mencionado en el apartado anterior, la causa del síndrome de Down es la presencia de un cromosoma extra en todas o en algunas de las células del individuo afectado. El proceso por el cual se llega a este resultado implica una serie de mecanismos complejos a los que nos referimos a continuación.

Todas las células que conforman al ser humano, a excepción de las sexuales, contienen 46 cromosomas, agrupados en 23 pares. De estos 23 pares, 22 están constituidos por autosomas y uno por cromosomas sexuales ( $X X$ en el caso de la mujer y $X Y$ en el caso del hombre). Los autosomas se ordenan en una serie según su longitud y se numeran del $1 \mathrm{al} 22$, yendo del más grande al más pequeño. La reproducción sexual tiene lugar debido a que las células sexuales, los óvulos y los espermatozoides, conllevan la mitad de información genética que contienen las restantes células corporales. Esta división del material genético ocurre durante la meiosis, proceso por el cual los cromosomas, dispuestos en parejas, se separan, yendo a parar cada miembro del par a diferentes células sexuales; por ello, tanto el óvulo como el espermatozoide contienen tan sólo 23 cromosomas (22 autosomas y un cromosoma sexual) en lugar de 46 . En el momento de la fecundación, la unión de las dos mitades repone la cantidad total de información genética y el huevo fertilizado de esta manera poseerá de nuevo un total de 46 cromosomas repartidos en 23 pares. A partir de este momento se irán sucediendo continuas divisiones celulares que darán como resultado células hijas con dotación cromosómica normal, hasta la formación completa del embrión.

No obstante lo anterior, hay ocasiones en las que este mecanismo falla y se producen errores en la escisión de los cromosomas. En el caso concreto del síndrome de Down, se observa que el par de autosomas 21 no se separa, resultando finalmente un par «trisómico», esto es, con tres cromosomas en lugar de dos. Tres son las condiciones en las que se presenta esta alteración causante del síndrome de Down: la trisomía regular, la trisomía en mosaico y la trisomía por traslocación. En las páginas siguientes se describe brevemente en qué consiste cada una de estas tres condiciones.

\section{Trisomía regular}

La trisomía regular es el caso más frecuente. Según Lambert y Rondal (1982), esta condición aparece en el $90 \%$ de los sujetos afectados por el síndrome de Down. En este caso concreto, el error de distribución de los cromosomas del par vigésimo primero se produce antes de la fertilización, o bien durante la primera división celular. 
Cuando el error tiene lugar antes de la fertilización, encontramos que una de las dos células sexuales es defectuosa. La Fig. 1 ilustra la distribución del cromosoma 21 a partir de un óvulo y un espermatozoide normales; en este caso el huevo fecundado poseerá dos cromosomas 21 , uno de cada padre, y las sucesivas divisiones de éste darán como resultado células hijas con dotación cromosómica normal para este par. La Fig. 2 (a) muestra lo que sucede cuando la distribución errónea del autosoma 21 ha tenido lugar durante la formación del óvulo o del espermatozoide, encontrándonos entonces con una alteración en uno

FIGURA I

Distribución normal del cromosoma 21.

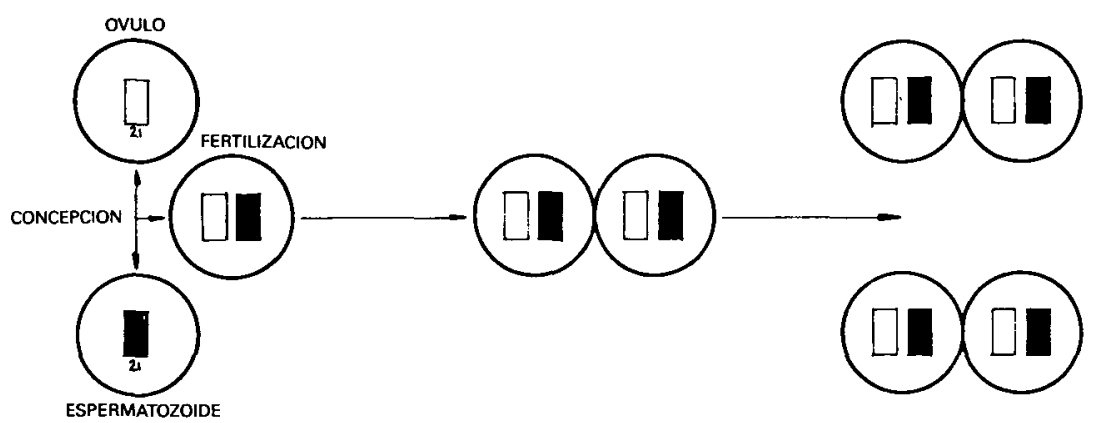

FIGURA II

Distribución errónea del cromosoma 21 en el caso de trisomía regular.

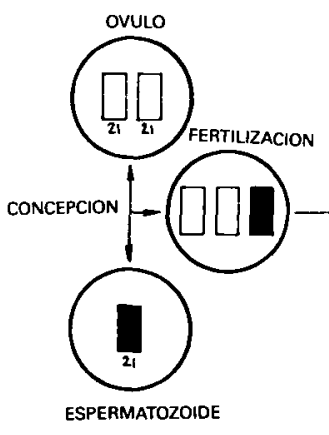

a) Antes de la fertilización

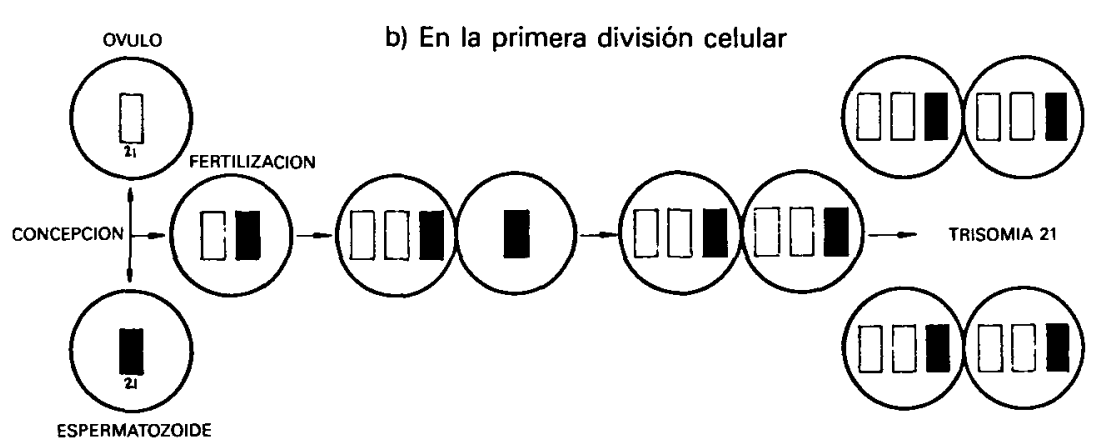


de los dos gametos; en dicha figura se observa que el óvulo contiene dos cromosomas 21 en lugar de uno, que es lo que debiera haber si no hubiese habido fallos en la repartición del material genético durante la meiosis. Este error de distribución, que puede afectar también al espermatozoide, hace que después de la concepción el huevo fertilizado contenga tres cromosomas 21, dos del gameto defectuoso y uno del gameto normal. Estos tres cromosomas se encontrarán en cada una de las células restantes de las divisiones posteriores, dando como resultado final un embrión con un cromosoma de más en el par 21 de todas sus células corporales.

La otra posibilidad es que la anomalía se produzca durante la primera división celular, de la forma en que queda ilustrado en la Fig. 2 (b). Aquí observamos que tanto el óvulo como el espermatozoide son normales y que la no escisión de los dos cromosomas del par 21 se produce durante la primera división celular de un huevo fecundado normalmente, que poseerá, por tanto, dos cromosomas 21. Cuando éste se divide por primera vez, encontramos, contrariamente a lo esperado, que las dos células hijas resultantes no presentan la misma dotación genética que la original para este par, sino que una de ellas ha recibido tres cromosomas 21, y la otra, uno sólo, en lugar de dos cada una. La célula con el cromosoma único no va a ser viable y el embrión se desarrollará exclusivamente a partir de la célula trisómica, presentando, como en el caso anterior, tres cromosomas 21 en todas sus célular corporales.

\section{Trisomía en mosaico}

El mosaico aparece en el 5\% de los sujetos afectados por el síndrome de Down (Lambert y Rondal, 1982). En este caso, el error de distribución de los cromosomas del par 21 se produce en la segunda o tercera división celular. En la Fig. 3 representamos un ejemplo de trisomía en mosaico, en el que la alteración se origina durante la segunda división celular.

Como queda reflejado en dicha figura, ahora tenemos un óvulo y un espermatozoide normales que presentan un sólo cromosoma 21. El huevo fecundado va a reunir estos dos cromosomas y va a contar con un par 21 dotado normalmente. Una vez ocurrida la primera división celular, tendremos dos células hijas con dotación también normal para este par. De estas dos células hijas, una se dividirá normalmente y la otra dará como resultado de su división otras dos cé-

\section{FIGURA III}

Distribución erónea del cromosoma 21 en el caso del mosaicismo.

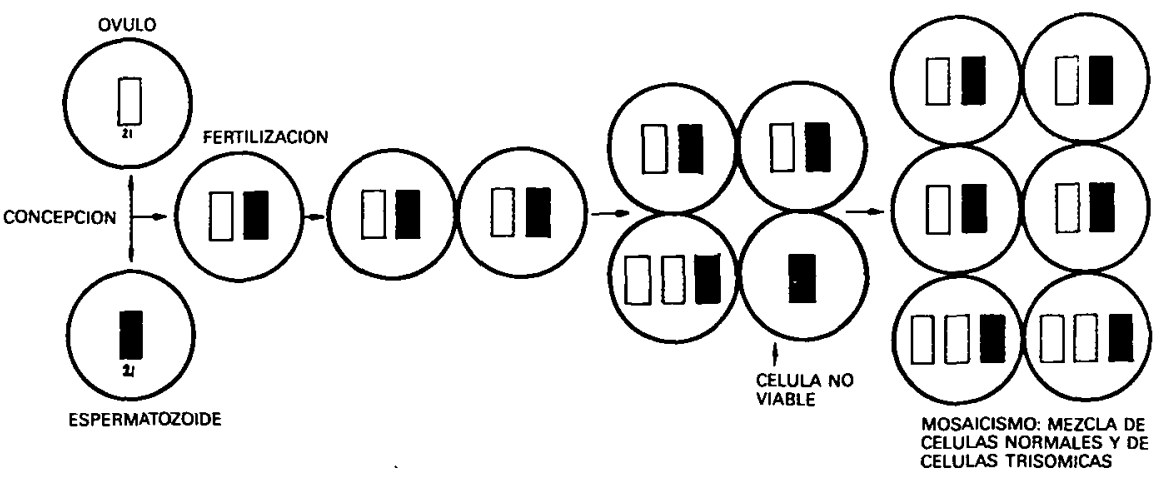


lulas con una distribución errónea del material cromosómico del par 21. En lugar de recibir cada una de ellas dos cromosomas 21, una recogerá tres y la otra tan sólo uno. De nuevo, esta última célula será inviable y el embrión se desarrollará a partir de una mezcla de células normales y trisómicas, con lo cual presentará la alteración en algunas de sus células corporales y no en todas. El número de ellas que resulten finalmente afectadas dependerá del momento en el que se produzca la división defectuosa; cuanto antes tenga lugar el incidente, más numerosas serán las células afectadas.

\section{Trisomía por traslocación}

La traslocación es la causa del síndrome de Down en el $5 \%$ restante de los casos (Lambert y Rondal, 1982). El témino traslocación hace referencia al hecho de que la totalidad o una parte de un cromosoma está unido a la totalidad o a parte de otro. Los cromosomas más afectados por esta alteración son los pares 13, 14, 15, 21 y 22. En la mayoría de los casos de traslocación, el error se produce en la formación del óvulo o del espematozoide, o tabién durante la primera división celular.

En el primer supuesto, el huevo fertilizado reunirá un cromosoma 21 del gameto normal y hasta dos del defectuoso, que se encontrarán distribuidos de la siguiente manera: uno estará libre en la célula y el otro, parcialmente o en su totalidad, adherido a otro cromosoma perteneciente a un par distinto del 21, como, por ejemplo, el 14. Cuando el embrión se desarrolle, todas sus células corporales contendrán, generalmente, 47 cromosomas (véase la Fig. 4).

FIGURA IV

Distribución errónea del cromosoma 21 por traslocación.

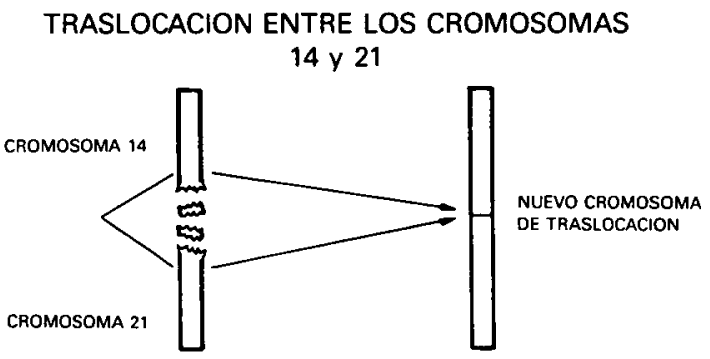

EJEMPLO DE TRASLOCACIÓN TRASMITIDA POR UNO DE LOS PADRES

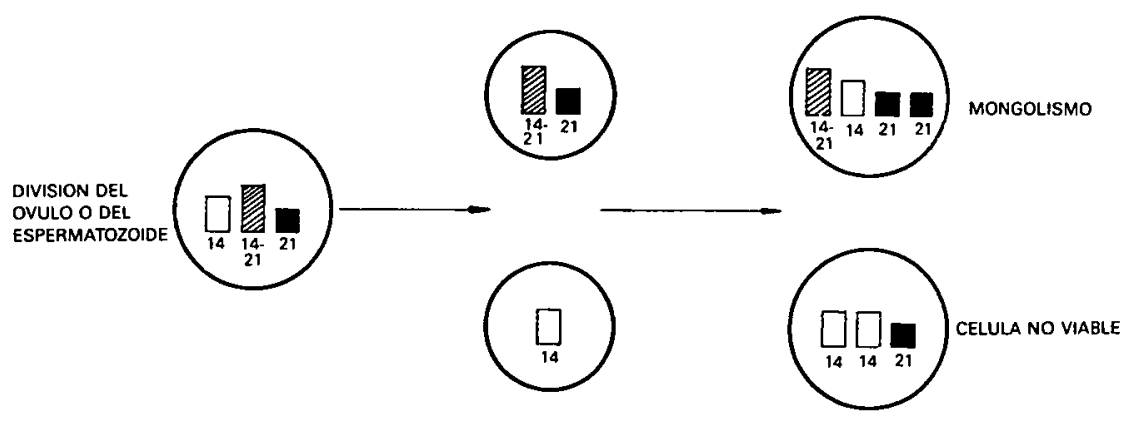




\section{4}

En el segundo supuesto de traslocación, la causa de la alteración sigue siendo la misma que en el anterior, sólo que el error en la distribución se produce durante la primera división celular de un huevo fecundado normalmente, que poseerá dos cromosomas de cada par. Las dos primeras células hijas no reproducirán la dotación genética de la original, sino que en el reparto cromosómico una recibirá tres cromosomas 21 (dos libres y el tercero adherido a un cromosoma de otro par), mientras que la otra recibirá un solo cromosoma del par 21. Esta última célula, monosómica para este par, no va a ser viable, así que, igual que en el supuesto anterior, el embrión se desarrollará a partir de una célula con tres cromosomas 21 presentando esta misma alteración genética en todas sus células corporales.

En un número muy reducido de casos, esta alteración no se produce ni en la formación del óvulo o del espermatozoide, ni durante la primera o segunda división celular. Este tercer supuesto implica que uno de los dos padres es portador de la traslocación. El padre portador será normal física e intelectualmente y presentará 46 cromosomas en todas sus células corporales, pero distribuidos de una forma errónea, que consiste en poseer un cromosoma 21 libre y otro adherido a un par distinto. Tras la fertilización, en ciertos casos, todas las células del embrión contendrán tres cromosomas 21 , dos del progenitor portador y el tercero del normal.

\section{Diferencias entre la trisomía 21 y otras trisomías cromosómicas.}

Ante un caso de síndrome de Down, sólo es posible saber si se trata de una trisomía regular, de un mosaico o de una traslocación mediante un minucioso análisis del material cromosómico del sujeto afectado. Aun así, en el caso de la trisomía regular y en el de la traslocación sin padres portadores, no podemos llegar a saber si la alteración se produjo antes de la fecundación, por defecto de alguno de los gametos sexuales o durante la primera división celular.

Tampoco conocemos en la actualidad por qué en la especie humana el par de cromosomas vigésimo primero sufre estas alteraciones con una frecuencia considerablemente alta. Según la mayoría de los autores (véanse, por ejemplo, Lambert y Rondal, 1982; Plomin, DeFries y McClearn, 1984), la incidencia del síndrome de Down es de un caso por cada 600-700 nacimientos de niños normales, cuando, de acuerdo con Plomin, DeFries y McClearn (1984), la incidencia de otras formas de trisomía, como la de los pares 13 ó 18, es de uno por cada 6.000 sujetos normales y uno por cada 5.000 , respectivamente.

Algunos autores han indicado que este hecho puede deberse a que los cromosomas del par 21 son relativamente pequeños comparados con los de los otros pares, de forma que las células soportarían mejor esta forma de trisomía que cualquiera otra que implicase un número más elevado de alteraciones al afectar una cantidad mayor de material genético (Cuilleret, 1985). Además, parecen existir una serie de factores que intervienen de algún modo en la aparición de esta patología, a los que haremos mención más adelante.

\section{Ejemplos no bumanos de trisomía cromosómica.}

Para finalizar con las bases genéticas del síndrome de Down, queremos indicar que se han encontrado también casos de trisomías cromosómicas en otros seres vivientes, además del hombre. Así, Smith y Berg (1978) destacan hallazgos como el de Blakeslee (1923), quien describió la existencia en ciertas plantas 
de doce procesos trisómicos, uno por cada par de cromosomas, demostrando que en este caso las modificaciones producidad no eran necesariamente nocivas; el de Morgan y cols. (1925), quienes encontraron que, en la Drosophila melanogaster, la trisomía de un pequeño cromosoma, el cuarto, producía una ligera disminución en el tamaño de la mosca, pero sin otras alteraciones, o el de Cattanach (1964), que describió un ratón de aspecto normal pero estéril, con trisomía de uno de los autosomas menores. Por su parte, McClure y cols. (1969) descubrieron una chimpancé hembra con trisomía de un pequeño cromosoma, designado con el número 22, que mostraba, entre otras alteraciones, un crecimiento físico lento, un desarrollo motor retardado, hipotonía muscular, defectos cardiacos congénitos y el epicanto muy pronunciado.

A pesar de todos estos hallazgos, el estudio de las trisomías que aparecen de forma espontánea en animales y plantas no ha proporcionado hasta el momento actual ninguna aclaración para comprender el fenómeno tal y como éste se da en la especie humana, por lo que intentar establecer algún tipo de comparación entre los distintos ejemplos indicados resulta prácticamente imposible. No obstante, como se verá más adelante, actualmente se está ensayando la inducción experimental de un estado de trisomía en el cromosoma 16 del ratón, que contiene al menos cuatro de los genes existentes en el cromosoma 21 humano, y cuyo estudio podría tener importantes implicaciones para la comprensión del síndrome de Down.

\section{FACTORES ASOCIADOS AL ORIGEN DEL SINDROME DE DOWN}

Los autores, como ya se ha mencionado previamente, coinciden en señalar la existencia de una serie de circunstancias o factores que, en mayor o menor medida, aparecen asociados al origen del síndrome de Down. Dichos factores se agrupan en las dos grandes categorías siguientes: factores intrínsecos y factores extrínsecos. A continuación se detallan las variables comprendidas en cada grupo.

\section{Factores intrínsecos}

Se consideran detro de este grupo los factores hereditarios y los relacionados con la edad de la madre. Dentro de los factores hereditarios se contemplan los casos siguientes:

a) Sujetos con sindrome de Down nacidos de madres afectadas por este mismo sindrome.

Debe destacarse que la probabilidad de que una madre afectada de síndrome de Down tenga hijos que presenten la misma alteración es de un $50 \%$.

b) Sujetos afectados en cuya familia aparece algún otro individuo con el sindrome de Down.

Son casos muy poco frecuentes. Si los sujetos afectados son hijos de padres normales, es muy difícil conocer las causas exactas de la existencia de más de un sujeto de la familia con la alteración. La única posibilidad que actualmente se ofrece a estas familias es la detección prenatal de nuevos casos.

c) Los casos de padres portadores de una translocación.

Según Lambert y Rondal (1982), sólo en el uno o dos por ciento de los afectados por el síndrome de Down puede demostrarse la existencia de una traslo- 
cación que afecte a los cromosomas del par 21 , ya provenga del material cromosómico del padre o de la madre, siendo excepcional que las situaciones de traslocación se encuentren a lo largo de una descendencia familiar. Como señalamos al revisar las alteraciones cromosómicas implicadas en el origen del síndrome de Down, la traslocación generalmente se produce en momentos muy determinados (en la formación del óvulo o del espermatozoide o durante la primera división celular) y siempre es impredecible.

\section{d) Los casos de padres mosaicos.}

Algunas veces sucede que uno de los padres es intelectual y físicamente normal (exceptuando algunos rasgos menores, tales como alteraciones en los pliegues palmares) a pesar de poseer una estructura cromosómica en mosaico. Como comentamos previamente, un individuo de estas características presenta una parte de sus células con 46 cromosomas y otra con 47 . En el caso que nos concierne, el padre afectado va a tener la mayoría de sus células normales y sólo una pequeña parte de ellas con un cromosoma extra perteneciente al par 21; estas células alteradas se van a encontrar principalmente en los testículos o en los ovarios. De todas formas, no existe un acuerdo unánime entre los investigadores acerca de si estas personas pueden ser transmisoras directas de cualquier alteración cromosómica, incluida la trisomía del par 21. Tampoco se ha demostrado que esta particularidad genética sea más frecuente entre los padres de hijos con síndrome de Down que entre los padres de hijos normales.

\section{e) Edad materna.}

Los otros factores intrínsecos que se consideran en la actualidad, como indicamos anteriormente, son los relacionados con la edad materna. Se ha observado desde hace mucho tiempo que el nacimiento de un niño con síndrome de Down es más frecuente a medida que aumenta la edad de la madre, sobre todo después de los 35 años. Lambert y Rondal (1982) indican que unos dos tercios de los niños con síndrome de Down nacen de madres con más de 30 años, y que la probabilidad de tener un hijo afectado aumenta con la edad hasta ser de uno por cada cincuenta después de los 40 años.

No sabemos aún a qué se debe esta supuesta relación entre la edad materna y la incidencia del síndrome de Down, aunque se piensa que en las mujeres de edad avanzada, debido al proceso normal de envejecimiento biológico de su sistema reproductor, se produciría una involución ovárica y una disminución de la capacidad reproductora, que podría explicar la mayor incidencia del síndrome de Down en su descendencia. Esta hipótesis no está demostrada en la actualidad, aunque sí se piensa que la combinación de distintos factores, entre los que se encuentran también los extrínsecos, que mencionamos a continuación, podrían favorecer este accidente genético (véase, por ejemplo, Wunderlich, 1977).

Con respecto al papel que la edad del padre pueda tener en el origen del síndrome de Down, los autores coinciden en señalar que es nulo o muy escaso, comparado con el que tiene la edad materna. Hasta el momento, los datos no aportan ningún tipo de correlación significativa entre esta variable y la aparición de descendientes con este síndrome. A un nivel explicativo muy general, se supone que el proceso de desarrollo de los gametos masculinos podría estar menos afectado por el envejecimiento biológico natural que el de los gametos femeninos (Smith y Berg, 1978). 
Factores extrinsecos.

Se recogen en este grupo aquellos factores que, por lo general, no implican de una manera tan estrecha como los intrínsecos la aparición de esta alteración genética, pero que, en un momento determinado, podrían actuar favoreciendo su causación. Las circunstancias que se contemplan dentro de este supuesto son las siguientes:

a) Los efectos de agentes mutágenos.

Se conoce desde hace tiempo que la actuación de diferentes agentes, tales como determinadas radiaciones, algunos compuestos químicos y ciertos virus favorecen la aparición de diversos tumores malignos, debido a su capacidad para inducir alteraciones en el material genético. Algunos autores se han planteado la influencia de tales factores para explicar el fenómeno de no disyunción de los cromosomas en el síndrome de Down. No obstante, es muy difícil verificar si existe una relación entre los diferentes agentes mutágenos y la trisomía 21, ya que la mayoría de las veces dichos agentes han intervenido en una época muy distante con respecto al momento preciso en el que se ha producido la alteración, siendo muy pocos los casos en los que podemos establecer con certeza una relación causal inmediata.

b) Los efectos de distintos trastomos orgánicos de los padres.

En algunos casos se ha puesto de manifiesto una posible conexión entre el síndrome de Down y determinados trastornos orgánicos de la madre o del padre. Entre tales trastornos se han destacado principalmente los siguientes: los desórdenes tiroideos maternos, especialmente el bocio tiroideo; los índices elevados de inmunoglobulina y de tireoglobulina en la sangre de la madre, que se incrementan conforme aumenta la edad de la misma, y las deficiencias vitamínicas maternas o paternas, especialmente la de vitamina $A$, que tiene efectos muy nocivos sobre el sistema nervioso, además de alterar el funcionamiento del organismo en general y contribuir a favorecer las alteraciones genéticas.

Para concluir con esta breve revisión de los factores que parecen estar asociados al origen del síndrome de Down, debe subrayarse que en la actualidad resulta imposible considerar cualquiera de los factores señalados como causas exclusivas de esta cromosomopatía. Es probable que todos ellos interactúen de forma múltiple y variada, y que sea esta interacción la que en definitiva pueda dar lugar a la aparición del síndrome de Down.

\section{ALTERACIONES ESPECIFICAS DEL SINDROME DE DOWN}

Las particularidades genéticas que definen el síndrome de Down tienen una manifestación fenotípica, que se traduce en un número elevado de anormalidades constitucionales y conductuales en la totalidad de los sujetos afectados en relación con los normales. Como señala Epstein (1986, 1987), a pesar de la existencia de notables diferencias individuales, los diversos autores consideran que las alteraciones clínicas más características que acompañan siempre al síndrome de Down son la hipotonía muscular y el retraso mental, desde momentos muy tempranos de la vida, así como también la aparición de un proceso de degeneración neuronal durante la edad adulta, análogo a la enfermedad de Alzheimer (demencia senil o presenil). Además, es sobradamente conocido que todos los 
sujeos afectados de este síndrome presentan, desde su nacimiento, anormalidades en su morfología corporal y, sobre todo, facial. A lo anterior hay que añadir la existencia, con una incidencia diversa, de numerosas alteraciones en la estructura y funcionamiento de los diferentes sistemas fisiológicos. Dichas alteraciones ocasionan importantes problemas de salud a los sujetos con trisomía 21 , dificultando su supervivencia, en especial durante los dos primeros años de vida.

A continuación revisamos, por este orden, los rasgos morfológicos que son más distintivos de la mayoría de los sujetos con síndrome de Down, centrándonos principalmente en la morfología facial y destacando aquellos aspectos que están presentes desde el nacimiento o que se hace evidentes durante los primeros meses de vida. Seguidamente, nos ocupamos del problema de la hipotonía muscular, del proceso de degeneración neuronal y de otros trastornos biomédicos que padecen estos sujetos, dedicando un último epígrafe al retraso mental.

\section{Morfologia de los individuos con sindrome de Down.}

De acuerdo.con los distintos autores (véase Wunderlich, 1977; Smith y Berg, 1978; Mendiguchia, 1980; Lambert y Rondal, 1982), debe destacarse que los sujetos con síndrome de Down tienen, salvo raras excepciones, una altura más reducida que la media. Aunque su tamaño al nacer es a menudo normal, antes de finalizar el primer año ya se puede observar una apariencia corporal rechoncha, que se hace más evidente con el transcurso del tiempo. Esto se debe fundamentalmente a la pequeña longitud de las extremidades inferiores y superiores en relación al tronco, que es de tamaño normal.

Los sujetos con trisomía 21 poseen, además, unas manos pequeñas y gruesas con unos dedos más cortos de lo normal, debido a que los huesos metacarpianos y las falanges tienen un tamaño notablemente reducido. Este hecho se acentúa en el caso del dedo meñique, lo que explica que la parte superior del mismo esté generalmente curvada hacia los otros dedos de la mano, provocando anomalías en su surco de flexión. Como se ilustra en la Fig. 5, a menudo la mano presenta sólo un pliegue palmar en lugar de dos. Por otra parte, los pies de estos sujetos son también cortos y anchos, destacando entre sus características más distintivas una separación notoria entre el primer y segundo dedo, de cuya hendidura nace un surco vertical que se extiende hasta ocupar parte de la región superior de la planta del pie (véase la Fig. 5).

Los sujetos afectados de este síndrome se destacan igualmente por poseer una cabeza, en perímetro y longitud, más pequeña de lo habitual. Su forma es excesivamente redonda y se caracteriza por un acortamiento del diámetro anteroposterior, un aplanamiento de la parte trasera u occipucio y una protuberancia occipital reducida o ausente. Las fontanelas suelen ser grandes y pueden cerrarse más tarde que en los niños normales; las suturas son anchas y la frontal puede extenderse hasta la glabela. Otro aspecto que debe subrayarse es que las orejas son cortas y estrechas, con lóbulos pequeños o ausentes, presentando, además, una superposición angular del hélix y un antihélix muy prominente (véase la Fig. 6). El cuello es, así mismo, corto y muy ancho, apariencia que se ve resaltada por el occipucio plano. Debe destacarse también que la piel aparece en la mayoría de los casos enrojecida o amoratada, haciéndose más gruesa y seca a medida que el niño crece. Por este motivo, se encuentra a menudo escamada y es muy vulnerable a distintos tipos de lesiones. Además, el cabello es generalmente escaso, fino y lacio. 
FIGURA V

Mano y pie característicos de los sujetos con sindmome de Down.
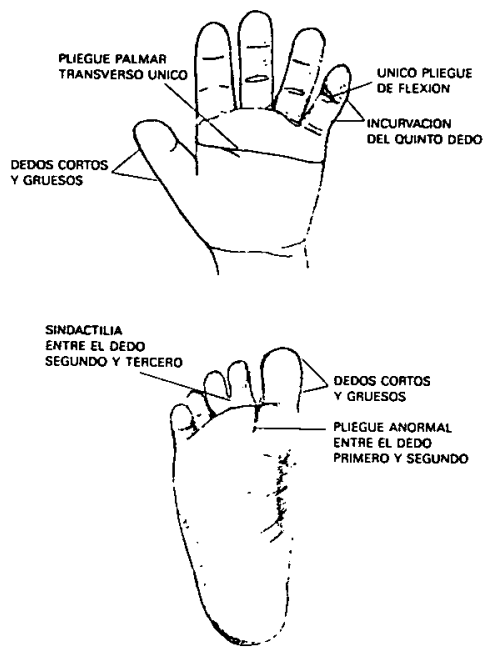

Aunque los rasgos descritos con anterioridad caracterizan por sí mismos esta alteración, la cara es, sin embargo, la parte que manifiesta las anomalías físicas más notables de entre todas las que definen el síndrome de Down. Esto es así hasta el extremo de que la mayoría de los sujetos afectados pueden reconocerse directamente por su apariencia facial, incluso desde los primeros momentos de la vida. Uno de los rasgos faciales que atraen inmediatamente la atención del observador es la morfología de los ojos. Como se puede apreciar en la Fig. 6, las fisuras palpebrales son, generalmente, oblicuas, y están estrechadas hacia fuera. La comisura externa está ligeramente más elevada que la interna, lo que favorece la configuración de unos ojos rasgados. La región epicántica presenta una pequeña capa de piel que nace de la región interna de la porción orbital y se conoce con el nombre de pliegue epicántico. Este último rasgo raramente se encuentra entre los sujetos europeos, si bien es muy frecuente en individuos de distintas poblaciones orientales, aunque con una apariencia ligeramente distinta de la que tiene en el síndrome de Down. Sin duda alguna, esta similitud morfológica constituyó uno de los motivos principales que llevaron a Down a considerar equivocadamente a los sujetos afectados por esta cromosomopatía como casos de regresión a la raza mongola.

Además de los ojos, también debe señalarse que la nariz es pequeña, con aplanamiento de la parte dorsal, asociado a un desarrollo incompleto e incluso ausencia de los huesos nasales. La porción cartilaginosa suele ser ancha y triangular, produciendo el efecto de una nariz chata. En la mayoría de los casos, las ventanas nasales apuntan hacia arriba en lugar de hacia abajo. Finalmente, la boca es también pequeña, y el maxilar superior aparece subdesarrollado. Los labios, en general, no presentan alteraciones morfológicas notables, pero sí la lengua, que es anormalmente grande. La pequeñez de la cavidad oral, y el débil tono muscular que acompaña al síndrome de Down, así como el tamaño excesivo de la lengua, hacen que ésta salga ligeramente de la boca con mucha frecuencia, siendo común en estos sujetos que la boca permanezca casi siempre abierta. 
FIGURA VI

Oreja y ojo de un sujeto normal y de un sujeto con sidrome de Down.
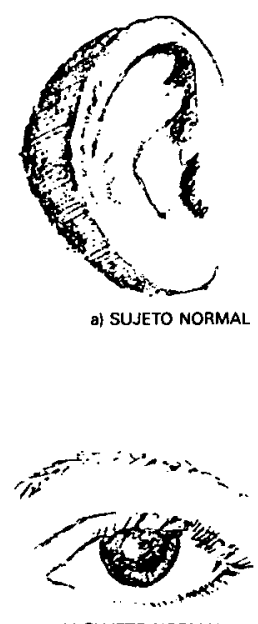

b) SUJETO NORMAL

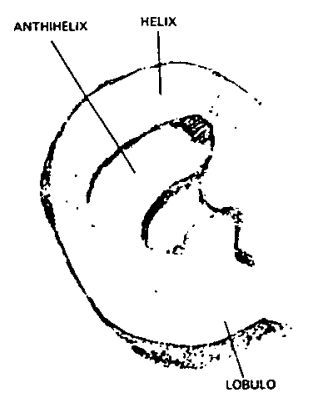

SUJETO CON SINDROME DE DOWN

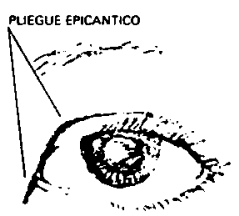

SUJETO CON SINDROME DE DOWN

\section{Hipotonía muscular y trastomos biomédicos.}

Todos los autores coinciden en señalar que los recién nacidos y niños pequeños con síndrome de Down muestran una hipotonía muscular generalizada, es decir, una insuficiencia en el mantenimiento y la organización del tono muscular. Dicha insuficiencia es especialmente notable durante los seis primeros meses de vida y tiende a hacerse menos pronunciada con el aumento de la edad (véase, por ejemplo, Cowie, 1970; Gibson, 1975; Smith y Berg, 1978; Morris y cols., 1982; Pueschel y Rynders, 1982; Scott y cols., 1983). Esta alteración en el tono muscular está relacionada en los sujetos con trisomía 21 con una respuesta deficiente a los estímulos, así como con numerosas anomalías psicomotrices, tales como una dificultad notable para realizar movimientos corporales, el retraso de la capacidad para sostener la cabeza, sentarse, permanecer de pie o andar, y, finalmente, diversas alteraciones en los reflejos.

En relación con este último aspecto, Cowie (1970) ha puesto de manifiesto que los reflejos palmar, plantar, de Moro y el de la marcha automática desaparecen más tarde en los bebés con síndrome de Down que en los niños normales. Así mismo, este autor observó un deterioro de la resistencia a la tracción de los brazos, una mala respuesta postural en una posición de suspensión ventral y una respuesta débil a la estimulación del reflejo patelar y de Moro. No obstante, como comentaremos más extensamente en un apartado posterior, hasta el momento aún se desconoce si las causas de la hipotonía muscular generalizada que se observa en el síndrome de Down y de sus manifestaciones asociadas reside en posibles anormalidades del músculo o en alteraciones en el desarrollo del sistema nervioso (véanse Smith y Berg, 1978; Epstein, 1986, 1987).

Con respecto a los diversos trastornos biomédicos que acompañan a esta anomalía cromosómica, destacamos en primer lugar el proceso de degeneración neuronal que aparece durante la edad adulta en los sujetos afectados. Sobre este aspecto, se sabe actualmente que todos los individuos con síndrome de Down 
que superan la edad de 35 años desarrollan en el cerebro el mismo tipo de placas seniles y de nudos neurofibrilares anormales que las personas que mueren por la enfermedad de Alzheimer, la forma más frecuente de demencia senil o presenil, y también que presentan el cuadro de deterioro cognitivo y emocional progresivo propio de los sujetos normales que padecen esta patología (Wisniewski y cols., 1978; Balazs y Brooksbank, 1985; Patterson, 1987).

Además de lo anterior, los sujetos trisómicos tienen más riesgo que los normales de sufrir determinados problemas de salud (véanse, por ejemplo, Smith y Berg, 1978; Lambert y Rondal, 1982; Pueschel y Rynders, 1982; Epstein, 1986; Patterson, 1987). Los más corrientes se derivan de la presencia de trastornos cardiacos e intestinales, a consecuencia de malformaciones congénitas del corazón y del tracto digestivo, y de la gran susceptibilidad que tienen al padecimiento de diversas infecciones, sobre todo respiratorias, debido a determinadas deficiencias de su sistema inmunológico.

También aparecen en el síndrome de Down, con una mayor frecuencia que en la población normal, trastornos sensoriales relacionados en particular con la visión y la audición. Así, se observa a menudo la presencia de estrabismo, que suele mejorar con el paso del tiempo, o de miopía y cataratas, siendo estas últimas bastante frecuentes en los sujetos de mayor edad. En cuanto a la capacidad de audición, ésta puede estar disminuida como resultado de diversas afecciones del oído medio, del oído interno y del nervio auditivo, ocasionadas generalmente por infecciones repetidas y por malformaciones anatómicas congénitas del aparato auditivo.

Por último, existen otros problemas severos de salud, tales como leucemia, diabetes o epilepsia, que también tienen una incidencia mayor en los sujetos con síndrome de Down que en los normales, pero que son menos usuales que los que hemos descrito con anterioridad (Smith y Berg, 1978; Epstein, 1986).

\section{Retraso mental.}

Es un hecho sobradamente conocido que los individuos con síndrome de Down presentan un cuadro importante de retraso mental, que se hace evidente desde edades muy tempranas. Tomando en consideración los estudios psicométricos, Lambert y Rondal (1982) han señalado que el cociente intelectual medio de los sujetos adultos afectados por este síndrome oscila entre 40 y 45 , pudiéndose alcanzar en casos muy aislados cocientes máximos de alrededor de 70 . Estos mismos autores han puesto de manifiesto que los sujetos con trisomía 21 experimentan un crecimiento mental hasta los 30 ó 35 años, aunque dicho crecimiento es muy lento a partir de los 15 años de edad.

Los investigadores se han planteado desde hace tiempo la posibilidad de que el cociente intelectual pueda variar en función de las diferentes categorías etiológicas de este síndrome, es decir, dependiendo de que el cuadro final esté determinado por un caso de trisomía regular, de mosaicismo o de traslocación. Los estudios que se han realizado al respecto arrojan resultados muy contradictorios y en la actualidad no puede extraerse ninguna conclusión definitiva, salvo indicar que los sujetos con trisomía 21 por mosaico tienen una mayor heterogeneidad genotípica, y, por tanto, también fenotípica, que los que presentan trisomía regular o traslocación, por lo que hay que esperar que sea en estos casos donde la variabilidad en relación con el rendimiento intelectual, entre otros aspectos, sea mayor (véase Gibson, 1973). 
En este contexto es casi innecesario señalar que el desarrollo cognitivo de los sujetos con síndrome de Down es, en general, mucho más lento que el de los sujetos normales, estando acompañado además de numerosos déficits en todos los procesos superiores. Por citar algunos aspectos más concretos, se conoce que los individuos trisómicos tienen disminuida su capacidad de discriminación visual y auditiva; muestran problemas importantes de atención, memoria a corto y largo plazo; dificultades en los procesos de aprendizaje, generalización y solución de problemas, y grandes limitaciones en la comprensión y producción del lenguaje verbal (véanse, por ejemplo, Borkowski y Wanschura, 1974; Furby, 1974; Gibson, 1975; Lambert y Rondal, 1982).

Si bien la mayoría de estos déficits se encuentran también en otros grupos de retrasados mentales, Gibson (1975) ha señalado que existen algunos aspectos que son específicos de los sujetos con trisomía 21 y que deben ser tenidos en cuenta por los investigadores. En este mismo sentido, Belmont (1971) puso de manifiesto que estos sujetos tenían más dificultades que otros retrasados para procesar información auditiva, pero que superaban a los demás en la ejecución de tareas relacionadas con el aprendizaje y el procesamiento visual de la información, canal, que, según este mismo autor, es el menos deteriorado en el síndrome de Down.

Por último, algunos autores se preocupan actualmente de relacionan las deficiencias observadas en la capacidad de procesamiento de información de los sujetos con trisomía 21 con la habituación más lenta de su respuesta de orientación en comparación con los sujetos normales. Con ello se han abierto nuevas líneas de investigación dentro de este campo de conocimiento, desarrolladas principalmente por psicofisiólogos y orientadas a esclarecer la naturaleza y dimensiones particulares del retraso mental en el síndrome de Down (Clausen y Sersen, 1983; Lincoln y cols., 1985).

\section{MECANISMOS NEURALES}

Las anormalidades clínicas más características del síndrome de Down son, como ya hemos indicado, el retraso mental, la hipotonía muscular y la aparición de un proceso de degeneración neuronal durante la edad adulta. Epstein (1986, 1987) apunta la posibilidad de que todas estas alteraciones se deban a la existencia de determinados trastornos neuroanatómicos o neurofisiológicos, los cuales, a su vez, deriven directamente del desarrollo de un estado de desequilibrio cromosómico. A continuación se detallan las anomalías neuroanatómicas y las disfunciones neurofisiológicas que parecen estar relacionadas con la trisomía 21, para terminar indicando las nuevas perspectivas de estudio existentes actualmente en este campo.

\section{Neuroanatomía.}

Antes de exponer los hallazgos más destacados en esta área, es preciso subrayar, primero, que a pesar de haberse identificado en diversas investigaciones determinadas patologías en el sistema nervioso central de los sujetos con trisomía 21, aún resulta imposible determinar la frecuencia y naturaleza de las mismas; y, segundo, que dichas investigaciones no permiten, por ahora, concluir la existencia de alteraciones neuroanatómicas específicas del síndrome de Down que puedan ser responsables de sus manifestaciones clínicas (véanse, por ejemplo, Gullotta y cols., 1981; Pueschel y Rynders, 1982; Balazs y Brooksbank, 1985). 
Teniendo en cuenta lo anterior, Scott y cols. (1983), una vez revisados los estudios existentes sobre esta materia, señalan que los niños con trisomía 21 muestran, principalmente, las siguientes anomalías neuroanatómicas: su cerebro pesa menos de lo normal; el cerebelo y el tronco del encéfalo están notablemente reducidos de tamaño; el diámetro fronto-occipital es menor de lo habitual, lo mismo que el tamaño de los lóbulos frontales, y, por último, el giro temporal superior resulta ser más estrecho de lo normal. Además de lo anterior, Sylvester (1983) ha observado recientemente en un feto la existencia de un desarrollo retardado del giro dentado y del hipocampo, presumiblemente relacionado con el menor tamaño de estas estructuras en el cerebro de los sujetos trisómicos adultos.

Marín-Padilla (1972), Suetsugu y Mehraein (1980) y Takashima y cols. (1981), por citar los autores más significativos, destacan la existencia de otras alteraciones estructurales en el cerebro de los niños con síndrome de Down. Dichos autores subrayan que, si bien el desarrollo de las dendritas y de las espinas dendríticas es normal durante la gestación, en los primeros momentos del período postnatal se aprecia ya una disminución en el número de espinas, las cuales parecen ser incluso más delgadas y cortas de lo usual. Por su parte, Wisniewski y Quinn (1984) indican que, muy poco tiempo después del nacimiento, se verifica también una reducción en la densidad de neuronas en determinadas capas del córtex. Finalmente, otros autores han puesto de relieve la existencia de una disminución del número de contactos sinápticos en cerebros pertenecientes a fetos y niños con trisomía 21 de hasta un año de vida (véase, por ejemplo, Scott y cols., 1983).

En general, teniendo en cuenta las consideraciones hechas al inicio de este apartado, la mayoría de los autores interpreta que el conjunto de las alteraciones observadas son probablemente la consecuencia de un proceso de maduración retardado y quizás detenido en algún punto, que se hace notar desde momentos muy tempranos de la vida, destacando la posibilidad de que sean motivadas por un proceso de diferenciación neuronal anómalo en el sistema nervioso del sujeto trisómico (véanse, por ejemplo, Takashima y cols., 1981; Epstein, 1986, 1987).

\section{Neurofisiología.}

Los cambios neuroanatómicos descritos en el apartado anterior, tales como el escaso número y reducido tamaño de las espinas dendríticas, no sólo parecen ser inespecíficos del síndrome de Down, sino que además se manifiestan fundamentalmente después del nacimiento. Varios autores consideran por ello que dichos cambios pueden ser secundarios a una disfunción neurofisiológica característica del estado de trisomía 21 . Sin embargo, como se verá a continuación, los conocimientos actuales acerca de esta hipótesis son también insuficientes, no habiéndose observado hasta este momento la existencia de anomalías neurofisiológicas específicas en el síndrome de Down que puedan dar cuenta de sus principales manifestaciones clínicas, esto es, de la hipotonía muscular y del retraso mental.

En el caso particular de la hipotonía, algunos autores la atribuyen a posibles anormalidades estructurales de las fibras musculares más que a una disfunción nerofisiológica central, en este último caso relacionada con las alteraciones observadas en el cerebelo de los sujetos con trisomía 21. En favor de esta posición periferialista, Landing y Shankle (1982) indican que las fibras de la musculatura esquelética de los sujetos con síndrome de Down presentan un diámetro mayor en relación con las de los sujetos normales, así como también un número redu- 
cido de núcleos por unidad de longitud, si bien los mismos autores reconocen que estos datos no son concluyentes para determinar si la hipotonía tiene una base central o periférica.

Otras explicaciones neurofisiológicas alternativas, que intentan dar cuenta del conjunto de manifestaciones clínicas que acompañan al síndrome de Down, se basan en el descubrimiento de disfunciones en los neurotransmisores periféricos. Así, por una parte, algunos autores se han basado en la hipersensibilidad de los sujetos con trisomía 21 a los efectos periféricos de la atropina para poner de relieve la existencia de una posible deficiencia constitucional en el sistema colinérgico de dichos sujetos, debida probablemente a una síntesis o liberación más lenta de acetilcolina (véase, por ejemplo, Lejeune, 1979); debe decirse, no obstante, que si bien la hipersensibilidad a los efectos midriáticos de la atropina resulta ser un dato consistente, existen resultados contradictorios acerca de sus efectos cardio-aceleratorios. Por otra parte, varios autores han señalado también la existencia de anomalías en el sistema adrenérgico; en particular, parece que la enzima dopamina-beta-hydroxilasa, que convierte la dopamina en noradrenalina, se encuentra en cantidades significativamente menores de lo normal en el plasma de los sujetos con trisomía 21.

A pesar de que los datos que acaban de presentarse sugieren la existencia de anomalías en el funcionamiento del sistema nervioso simpático y del parasimpático, hay que subrayar que todavía se ignora cuál es la naturaleza de las mismas (véanse, Weinshilboum y cols., 1971; Lake y cols., 1979). Para resolver esta cuestión, es preciso conocer si existen anormalidades en el sistema nervioso central semejantes a las encontradas en los neurotrasmisores periféricos; en este sentido, aunque se ha observado que las disfunciones del sistema colinérgico descritas con anterioridad aparecen también en las estructuras nerviosas centrales de los sujetos adultos con síndrome de Down, se desconoce si tales disfunciones u otras distintas están ya presentes en los niños de corta edad y, en consecuencia, si tienen algo que ver con el desarrollo del retraso mental. Como han señalado diversos autores, de comprobarse esto último quedaría plenamente justificada la intervención farmacológica precoz para afrontar el tratamiento del retraso mental. En la actualidad, si bien se conoce que la administración de 5-hidroxitriptamina no afecta al desarrollo intelectual, todavía se ignoran las posibilidades terapéuticas que ofrecen otros agentes químicos (Weise y cols., 1974; Share, 1976).

También se ha pueste de manifiesto la existencia de posibles alteraciones electrofisiólogicas en los sujetos trisómicos. No obstante, distintos autores, después de analizar los registros electroencefalográficos de estos sujetos en distintas condiciones, no han podido concluir la existencia de un patrón de actividad electroencefalográfica específico de la trisomía 21, aunque parece ser que los contactos sinápticos en distintas poblaciones neuronales corticales se encuentran empobrecidos (véanse, Ellingson y cols., 1973; Elul y cols., 1975) y también que existe un ligero aumento de la actividad theta (Tangye, 1979). A pesar de que, recientemente, se ha dado cuenta de la existencia de anormalidades en los potenciales evocados visuales y auditivos de los sujetos con síndrome de Down, en relación con los pertenecientes a otros grupos de retrasados mentales y de sujetos normales (véanse, por ejemplo, Gliddon y cols., 1975; Squire y cols., 1980), todos estos hallazgos se relacionan con los descritos anteriormente acerca de las deficiencias estructurales de las dendritas, interpretándose que tan sólo constituyen el reflejo de un estado de inmadurez cerebral. 
Por su parte, Scott y cols. $(1982,1983)$ han observado alteraciones bioeléctricas en neuronas cultivadas in vitro, pertenecientes al ganglio de la raíz dorsal de fetos con trisomía 21, cuya edad gestacional estaba comprendida entre las 16 y las 23 semanas. En particular, estos autores señalan que la tasa de conducción del potencial de acción a través de las dendritas es un $15.5 \%$ inferior de lo normal. A pesar de la escasez de datos existentes, Epstein (1986) destaca la importancia de continuar con el estudio de cultivos celulares in vitro, en especial de neuronas del sistema nervioso central, para dilucidar los efectos de la trisomía sobre la función neural.

Por último, dado que los estudios electrofisiológicos que acaban de mencionarse son todavía escasos y poco concluyentes, y puesto que el conjunto de hallazgos neurofisiológicos mencionados hasta ahora no sustentan la hipótesis de una disfunción específica de la trisomía 21, también algún autor se ha planteado la posibilidad de que exista alguna anomalía bioquímica de carácter molecular que pueda dar cuenta de la totalidad de las alteraciones neurobiológicas y conductuales asociadas al síndrome de Down. Así, por ejemplo, se ha llamado la atención sobre el hecho de que los cerebros correspondientes a varios sujetos con trisomía 21, de edades comprendidas entre 18 y 50 años, presentan cantidades reducidas de mielina, y también que todos lo fosfolípidos de la misma contienen una menor cantidad de ácidos grasos monoinsaturados que la de los sujetos normales. Si bien dichos cambios pueden afectar a la permeabilidad de la membrana y a los procesos de transporte neuronal, todavía se desconoce su naturaleza, por lo que una vez más no sabemos si se trata de la causa o el efecto de una anomalía estructural más general que afecte al crecimiento neuronal y a las sinaptogénesis (en relación con este aspecto, véase, Shah, 1979). De acuerdo con esto último, Lejeune (1979) ha sugerido que la totalidad de las alteraciones descritas podrían tener en último término un origen metabólico. Sin embargo, aunque esta hipótesis debe ser tenida en cuenta, todavía no se dispone de datos suficientes como para poder considerarla una explicación unitaria de la patogénesis propia del síndrome de Down, siendo preciso esclarecer en futuras investigaciones qué posibles alteraciones metabólicas subyacen a las disfunciones neurofisiológicas aquí descritas.

Nuevas perpectivas de estudio.

Como se ha venido señalando en los puntos que integran esta revisión, los datos disponibles hasta ahora no sustentan la idea de una alteración neuroanatómica o neurofisiológica específica del síndrome de Down, por lo que empieza a cuestionarse la existencia de un único factor causal de las manifestaciones clínicas asociadas a este síndrome, a la vez que se apunta una explicación multivariada, considerando sobre todo la interacción entre las distintas anomalías fisiológicas que parecen existir en el metabolismo, las propiedades eléctricas de la membrana y la síntesis y liberación de neurotransmisores. Puesto que tales disfunciones no parecen estar relacionadas con defectos neuroanatómicos, resulta de un enorme interés en estos momentos el desarrollo de estrategisas terapéuticas de atención precoz, ya sean éstas biológicas o conductuales, destinadas a mejorar la función del sistema nervioso central en los sujetos con trisomía 21.

Desde un punto de vista teórico, la clave del problema está en saber cuál es la relación existente entre la presencia de un cromosoma 21 extra y las distintas manifestaciones fenotípicas del síndrome de Down, teniendo en cuenta que esta dotación extranumeraria se traduce en un aumento del $50 \%$ en la expresión de los genes activos de ese cromosoma. En otros términos, es necesario 
localizar e identificar los genes presentes en el cromosoma 21 o, lo que es lo mismo, debe trazarse su mapa genético. Esto último implica averiguar cuáles de esos genes son activos, de qué fenotipos son responsables y qué consecuencias se derivan de su sobreactividad.

En la actualidad, se sabe que de los 100.000 mil genes funcionales que posee el ser humano, aproximadamente 1.500 se encuentran en el cromosoma 21, de los cuales, hasta el momento, sólo se han podido identificar alrededor de 20. Uno de dichos genes es el denominado gen Gart, el cual codifica tres enzimas diferentes implicados en la síntesis de purinas. Como señala Patterson (1987), a partir de este descubrimiento se ha podido saber por qué los sujetos con síndrome de Down presentan niveles altos de purinas en el suero, y, yendo aún más lejos, si tenemos en cuenta que dichos niveles alterados de purinas en el suero parecen estar relacionados con una amplia variedad de trastornos, incluido el retraso mental, se ha apuntado que la trisomía del gen Gart pudiera ser razón suficiente para causar alguna de las alteraciones clínicas que parecen ser propias del síndrome de Down. Si esto último fuera cierto, el procedimiento terapéutico más idóneo para tratar dichas manifestaciones clínicas consistiría en restablecer los niveles normales de purinas mediante el control de la expresión de este gen en fases tempranas del desarrollo fetal. Algo parecido podría decirse acerca de la mayor susceptibilidad de los sujetos con trisomía 21 a padecer leucemia, lo cual podría deberse a una alteración en la actividad de uno o más oncogenes localizados en el cromosoma 21. En relación con lo anterior, dado que al menos cuatro de los genes que se encuentran en el cromosoma 21 humano también están presentes en el cromosoma 16 del ratón, la inducción de un estado de trisomía 16 total o parcial en el ratón se presenta hoy como un modelo experimental de la trisomía 21 humana, apropiado para la realización de estudios neuroanatómicos, neurofisiológicos y neuroquímicos, in vivo y también in vitro (una exposición más extensa y detallada sobre esta cuestión puede verse en Epstein, 1986).

Para concluir con esta revisión, queremos resaltar que esta nueva aproximación al estudio del síndrome de Down, apenas iniciada, constituye, sin duda, la vía más idónea para conocer en el futuro no sólo cómo la trisomía del cromosoma 21 se traduce en un gran número de alteraciones fenotípicas en los sujetos afectados, sino también qué es lo que se puede hacer para neutralizar o mejorar tales alteraciones. No cabe duda de que ello constituye el gran reto a afrontar en los próximos años por los científicos interesados en el estudio del síndrome de Down.

\section{Referencias}

BaLAzs, R, y BRoOKSBAND, B. W. (1985): Neurochemical approaches to the paghogenesis of Down's syndrome. Joumal of Mental Deficiency Research, 29 (1), 1-14.

Belmont, J. H. (1971): Medical behavioural research in retardation. En N. R. Ellis (Ed.): International review of research in mental retardation (Vol. 5). Nueva York: Academic Press.

BLAKESLEE, A. F. (1923): Variations in the Jimson weed (Datura stranmonium) caused by differences in the number of chromosomes. Citado en J. F. Smith y J. M. Berg (1978): Sindrome de Down (mongolismo). Barcelona: Editorial Médica y Técnica (traducción española de la segunda edicción inglesa de 1976).

BorkowsKI, J. y W ANSCHURA, P. (1974): Mediational processes in the retarded. En N. R. Ellis (Ed.): Intemational review of mental retardation (Vol. 7). Nueva York: Academic Press.

Cafferata, J. F. (1909): Contribution à la littérature du mongolisme. Citado en G. F. Smith y J. M. Berg (1978): Síndrome de Down (mongolismo). Barcelona: Editorial Médica y Técnica (traducción española de la segunda edición inglesa de 1976). 
CAldeCotT, C. (1909): Tuberculosis as a cause of death in mongolism. British Medical Journal, 2, 665 .

Cattanach, B. M. (1964): Autosomal trisomy in the mouse. Cytogenetics, 3, 159.

CLARK, R. M. (1929): The mongol: A new explanation. Joumal of Mental Science, 75, 261.

Clausen, J. y Sersen, E. A. (1983): The orienting response and intellectual retardation. En D. Siddle (Ed.): Orienting and babituation: Perspectives in human research. Nueva York: John Wiley \& Sons.

CowIE, V. (1970): A study of the early development of mongols. Oxford: Pergamon Press.

Crookshanx, F. G. (1924): The mongol in our midst. Citado en G. F. Smith y J. M. Berg (1978): Sindrome de Down (mongolismo). Barcelona: Editorial Médica y Técnica (traducción española de la segunda edición inglesa de 1976).

Cuilleret, M. (1985): Los trisómicos entre nosotros. Barcelona: Masson.

Down, J. L. H. (1866): Observation on an ethnic classification of idiots. Clinical Lectures an Reports (Hospital de Londres), 3, 259.

ElLingson, R. J.; Eysen, J. D. y OtTersBerg, G. (1973): Clinical electroencephalography observation on institutionalized mongoloids confirmed by karyotype. Electroencephalography and Clinical Neurophysiology, 34, 193-196.

Elul, R.; Hanley, J. y Simmons, J. Q. III (1975): Non-Gaussian behavior of the EEG in Down's syndrome sugests decreased neuronal connections. Acta Neurológica Scandinavica, 51, 21-28.

Epstein, C. J. (1986): The consequences of chromosome imbalance: Principles, mechanisms and models. Nueva York: Cambridge University Press.

EPSTEIN, C. J. (1987): Down's syndrome. En G. Adelman (Ed.): Encyclopedia of Neuroscience (Vol. I). Boston: Birkhäuser.

Esquirol, J. E. D. (1938): Des maladies mentales considerés sous les repports médical, bygiénique et médico-légal (2 vols.). París: J. B. Baillière.

FurBY, L. (1974): Attentional habituation in mental retardation. Human Development, 1, $118-138$

GiBSON, D. (1973); Karyotype variation and behavior in Down's syndrome, Methodological review. American Journal of Mental Deficiency, 78, 128-183.

GiBson, D. (1975): Chromosomal psychology and Down's syndrome (mongolism). Canadian Journal of Behavioural Science, 7 (3), 167-191.

Gliddon, J. B.; Busk, J. y Galbraith, G. C. (1975): Visual evoked responses as a function of light intensity in Down's syndrome and non-retarded subjects. Psychopbysiology, 12, 416:422.

Gould, S. J. (1983): El pulgar del panda. Barcelona: Editorial Bruguera.

GullotTA, F.; ReHDER, H. y GROPP, A. (1981): Descriptive neuropathology of chromosomal disorders in man. Human Genetic, 57, 337-334.

Halbertsma, T. (1923): Mongolism in one of twins and the etiology of mongolism. Citado en G. F. Smith y J. M. Berg (1978): Sindrome de Down (mongolismo). Barcelona: Editorial Médica y Técnica (traducción española de la segunda edición inglesa de 1976).

JANSEN, M. (1921): Feebleness of growth and congenital dwarfism. Oxford: Medical Publications.

JONES, R. (1890): The mouth in backward children of mongolian type. Journal of Mental Science, 36, 187.

$\mathrm{KEAY}$, A. J. (1958): The significance of twins in mongolism in the light of new evidence. Joumal of Mental Deficiency Research, 2, 1.

Lake, C. R.; Ziegler, M. G.; Coleman, M. y Koping, I. J. (1979): Evaluation of the sympathetic nervous system in trysomy 21 (Down's syndrome). Journal of Psychyatric Research, 15 (1).

LAmbert, J. L. y Rondal, J. A. (1982): El mongolismo. Barcelona: Herder.

LANDING, B. H. y SHANKLE, W. (1982): Reduced number of skeletal muscle fiber nuclei in Down's syndrome: Speculation on a shut off" role of chromosome 21 in control of DNA and nuclear replication rater, possibly via determination of cell surface area per nucleus. Citado en C. J. Epstein (1986): The consequences of chromosome imbalance: Principles, mechanisms, and models. Nueva York: Cambridge University Press.

Lejeune, J. (1979): Investigations biochemiques et trisomie 21. Année Génétique, 22, 67-75.

Lejeune, J.; Turpin, R. y Gautier, M. (1959): Le mongolisme, premiere exemple d'aberration autosomique humaine. Année Génétique, 41-49.

Lelong, M.; Borniche, P; Kreisler, L. y Baudy, R. (1949): Mongolien issu de mére mongolienne. Archives Françaises Pediatric, 6, 231.

Lincoln, A. J.; Courchesne, E; Kilman, B. A. y Galambos, R. (1985): Neuropsychological correlates of information-processing by children with kown's syndrome. American Journal of Mental Deficiency, 89 (4), 403-414.

Marín.PAdilla, M. (1972): Structural abnormalities of the cerebral cortex in human chromosomal aberrations: A Golgi study. Brain Research, 44, 625-629.

McClure, H. N.; Belden, K. H.; Pieper, W. A. y Jacobson, C. B. (1969): Autosomal trisomy in a chimpanzee: Resemblance to Down's syndrome. Science, 165, 1.010. 
Mendiguchia, F. J. (1980): Psiquiatría infanto-juvenil. Madrid: Ediciones del Castillo.

Morgan, T. H.; Bridges, C. B. y Surtevant, A. H. (1925): The genetics of Drosophila. Bibliographical Genetica, 2,1.

Morris, A. F. V VuGhin, S. E. y Vaccaro, P. (1982): Measurements of neuromuscular tone and strength in Down's syndrome children. Joumal of Mental Deficiency Research, 26 (1), 41-46.

Oliver, C. A. (1891): A clinical study of the ocular symptons found in the so-called mongolian type of idiocy. Citado en G. F. Smith y J. M. Berg (1978): Sindrome de Down (mongolismo). Barcelona: Editorial Médica y Técnica (traducción española de la segunda edición inglesa de 1976).

Patterson, D. (1987): Las causas del síndrome de Down. Investigación y Ciencia, 133, 28-35.

Penrose, L. S. (1951): Maternal age in familial mongolism. Jourmal of Mental Science, 97, 738.

PenRose, L. S. (1953): Mongolian idiocy (mongolism) and maternal age. Annals of the Nueva York Academy of Sciences, 57, 494.

Plomin, R.; DeFries, J. C. y McClearn, G. E. (1984): Genética de la conducta. Madrid: Alianza.

PuesChel, S. M. y Rynders, J. E. (1982): Down's syndrome: Advances in biomedicine and the behavioral sciences. Cambridge: Ware Press.

ReHN, A. T. y ThOmAS, E. (1957): Family history of a mongolian girl who bore a mongolian child. American Journal of Mental Deficiency, 62, 496.

Reuben, M. S. y KleIN, S. (1926): Mongolian idiocy in both twins. Citado en G. F. Smith y J. M. Berg (1978): Síndrome de Down (mongolismo). Barcelona: Editorial Médica y Técnica (traducción española de la segunda edición inglesa de 1976).

Scott, B. S.; Becker, L. E. y Petit, T. L. (1983): Neurobiology of Down's syndrome. Progress in Neurobiology, 21, 199.237.

Scott, B. S.; Petti, T. L.; Becker, L. E. y Edwards, B. A. (1982): Abnormal electric menbrane properties of Down's syndrome DRG neurons in cell culture. Developmental Brain Reserch, 2 (2), 257-270.

SĖGUIN, E. (1846): Le traitement moral, l'bygiène et l'education des idiots. París: J. B. Baillière.

SHAH, S. N. (1979): Fatty acid composition of lipids on human brain myelin and synaptosomes: Changes in phenilketonuria and Down's syndrome. International Joumal of Biochemistry, $10,477-482$.

Share, J. B. (1976): Review of drug treatment for Down's syndrome persons. American Journal of mental Deficiency, 80 (4), 388-393.

Shuttleworth, G. E. (1906): Comment on R. Langdom Down's paper. Citado en G. F. Smith y J. M. Berg (1978): Síndrome de Down (mongolismo). Barcelona: Editorial Médica y Técnica (traducción española de la segunda edición inglesa de 1976).

SMITH, G. F. y BERG, J. M. (1978): Sindrome de Down. Barcelona: Editorial Médica y Técnica (traducción española de la segunda edición inglesa de 1976).

Souire, N.; Aine, C.; Buchwald, J.; Norman, R. y Galbraith, G. (1980): Auditory brain stem respones abnormalities in severely and profundly retarded adults. Electroencephalography and Clinical Neuropbysiology, 50, 172-185.

Suetsugu, M. y Mehraein, P. (1980): Spine distribution along the apical dendrites of the pyramidal neurons in Down's syndrome. Acta Neuropathologica, 50, 207-210.

Sutherland, G. A. (1899): Mongolian imbecility in infants. Practitioner, 63, 632.

SyLVESTER, P. E. (1983): The hippocampus in Down's syndrome. Joumal of Mental Deficiency Research, 27, 217.

Takashima, S.; Becker, L. E.; Armstrong, D. L. y Chan, F. (1981): Abnormal neuronal development in the visual cortex of the human fetus and infant with Down's syndrome: a quantitative and qualitative Golgi study. Brain Research, 225 (1), 1-21.

TANGYE, S. R. (1979): The EEG and incidence of epilepsy in Down's syndrome. Journal of Mental Deficiency Research, 23, 17-24.

TREDGOLD, A. F. (1908): Mental deficiency (amentia). Londres: J. B. Baillière, Tindall and Cox.

Turpin, R. y Caratzali, A. (1934): Remarques sur les ascendants et les collatéraux des sujets atteints de mongolisme. Citado en G. F. Smith y J. M. Berg (1978): Sindrome de Down (mongolismo). Barcelona: Editorial Médica y Técnica (traducción española de la segunda edición inglesa de 1976).

Weinshilboum, R. M.; ThoA, N. B.; Johnson, D. G..; Kopin, I. J. y Axelrod, J. (1971): Proportional release of norepinephrine and dopamine-beta-hydroxylase from sympathetic nerves. Science, 174, 1.349-1.351.

Weise, P.; Koch, R.; Shaw, K. N. F. y Rosenfeld, M. J. (1974). The use of 5-HTP in the treatment of Down's syndrome. Pediatrics, 54, 165-168.

WisNiewski, K.; Howe, J.; WILliams, D. G. y WIESNIEwSKI, M. M. (1978): Precocious aging and dementia in patients with Down's syndrome. Biological Psychiatry, 13 (5), 619-627.

WISNIEwSKI, K. y QUINN, M. R. (1984): Somatomedins and Down's syndrome. Biological Psychiatry, 19 (4), 469-470.

WunderLICH, C. (1977): The mongoloid child: Recognition and care. Tuason, A. Z.: U. Arizoma Press. 